\title{
'The Usual Suspects' but Always Ignored: The Nutritional and Immune System's Status Affect the Infective Endocarditis Hospital Mortality
}

\author{
Ozlem Arican Ozluk*, Ayse Akdeniz, Elif Guclu, Burcu Tuncay, Tahsin Bozat
}

Department of Cardiology, Bursa Ihtisas Training and Research Hospital, Bursa, Turkey

Email address:

oarican@yahoo.com (O. A. Ozluk)

${ }^{*}$ Corresponding author

\section{To cite this article:}

Ozlem Arican Ozluk, Ayse Akdeniz, Elif Guclu, Burcu Tuncay, Tahsin Bozat. 'The Usual Suspects' but Always Ignored: The Nutritional and Immune System's Status Affect the Infective Endocarditis Hospital Mortality. International Journal of Immunology.

Vol. 8, No. 1, 2020, pp. 9-12. doi: 10.11648/j.iji.20200801.12

Received: April 6, 2020; Accepted: May 5, 2020; Published: May 14, 2020

\begin{abstract}
The therapy of Infective Endocarditis is a very long and difficult. There are many factors that affected the management of therapy. Among them, maybe the most ignored factor belongs to the patient's' immune-nutrition status. Even though this assessment is more complicated; in recent years, prognostic nutritional index (PNI) was an established that inflammation-based nutritional score and the lymphocyte to-CRP ratio (LCR) was accepted as a marker of immune-inflammation's marker. Our study is aimed to investigate the relationship between LCR and PNI values and in-hospital mortality. Our comprehensive tertiary cardiology hospital in Turkey was included in this observational study, 36 patients with definite infective endocarditis were enrolled. We calculated LCR and PNI index from blood samples results of first hospitalization day. P-value $<0.05$ was considered as statistically significant. In hospital mortality occurred in $12(33 \%)$. LCR index was significantly lower in hospital mortality group $(\mathrm{p}=0.04)$ PNI index was also lower in hospital mortality group but it was not significant. The nutritional and immune system's status of the patient at the beginning of the treatment determines the hospital mortality of the patient during challenging treatment. In this context, the assessment of the PNI index and lymphocyte to-CRP ratio could aid physicians in determining hospital mortality risk and could affect the management of infective endocarditis patients.
\end{abstract}

Keywords: Endocarditis, Nutrition, Immune System

\section{Introduction}

Infective endocarditis (IE) is defined as an microbial infection of the endocardial surface of the heart, which may include one or more heart valves, mural endocardium or septal defect [1]. It is difficult to determine the exact incidence of IE worldwide, as the incidence numbers vary across countries [2, 3]. Between 2000 and 2011, the incidence of IE in the United States rose from 11 per 100,000 population to 15 per 100,000 population [4]. IE disease, the diagnosis and treatment is quite difficult even with today's knowledge and equipment, it is a long and high mortality treatment. The disease is a disease that affects all systems of the body beyond the infection of the disease, which is a simple cardiac involvement. Now, depending on the causative factor, even if the appropriate antibiotic treatment is applied, the response to the treatment also has a relationship with the patient's resistance and reservoir. However, there are not enough studies in the literature on this subject. This question comes in our mind, "why is the importance of nutritional and the immune system so neglected in the modern medical cardiology world'. This subject, which has not been given enough attention, is one of the most important contradictions that we physicians have in the treatment of today's infective endocarditis. In this study, we examined the relationship between Progression Nutrional Index and the Immune-Nutrional Index values, which have been emphasized in many studies, and the hospital mortality in our hospital, which serves as a Tertiary Cardiology Center. 


\section{Materials and Methods}

Between March 2016 and May 2019, 36 consecutive confirmed patients with IE, who were admitted to Bursa Yuksek Ihtisas Training and Research Hospital, were evaluated prospectively. Blood tests on the first day of hospitalization were taken into consideration for basic blood evaluations of the patients. Echocardiographic examination was performed in the first 48 hours of admission to the Cardiology unit with Transthoracic and, if necessary, Transesophageal examination. If needed serial echocardiographic evaluations were made. Blood cultures were taken as indicated in the guideline [5]. Infective Endocarditis was diagnosed based on ESC 2015 diagnostic criteria [5]. The microorganisms were identified from the blood cultures and cultures from the excised valve tissue. The study received approval from the ethics committee of Haydarpasa Training and Research Hospital Institution.

\subsection{Laboratory Measurements of PNI and LCR}

Peripheral blood samples from each patient were obtained within the first day of hospitalization. The whole blood cell count analysis were performed using Beckmann Coulter LH780 Hematology Analyzer device and Biochemical measurements were performed using Roche diagnostic Cobas 8000 (manheim). The LCR was calculated as follows: Lymphocyte count (number/mL)/CRP level (mg/dL). PNI was also calculated according to the formula: $10 \mathrm{x} \mathrm{Alb}+0.005 \mathrm{x}$ total lymphocyte count (as previously described) [6-8].

\subsection{Statistical Analysis}

Continuous data studies were presented as the mean \pm standard deviation. Categoric variables were presented as the number and percentage. The difference between the groups with respect to continuous variables were tested with Student's t-test or the Mann-Whitney U test, with the former being used for normally distributed variables and the latter for non-normally distributed ones. The difference with regard to categoric variables were tested using the chi-squared test. All statistical analysis were performed using SPSS package for Windows version 21 (SPSS Inc., Chicago, IL, USA). For all statistical analysis, p-value of $<0.05$ was considered statistically significant.

\section{Results}

The overall study consisted of 36 patients with an average age of $56 \pm 16$ years, of whom 24 were male and 12 were female. In-hospital deaths occurred in 12 patients (33\%). These patients, who died in the hospital are named as Group II, were significantly older than the others. $22 \%$ of the patients (n $=8$ ) had no high fever and the rest had a high fever. Both groups were similar in terms of basic parameters of their hemoglobin, white blood cells and platelets levels. But when we look at the distribution of the white blood cells, there were significant differences between the lymphocytes count in both groups. Additionally, urea and serum creatinine levels were significantly higher in group II $(\mathrm{p}<0.05)$. There were significant differences between Group I and Group II with respect to LV ejection fraction and LV systolic - diastolic diameters. The basic blood tests and echocardiographic findings of the patients are summarized in Table 1. Only $38 \%$ of our infective Endocarditis patients $(n=14)$ had detected positive blood cultures. These blood culture were positive in 6 of 12 patients who had an in-hospital death. All patients had major echocardiographic endocarditis findings. The distribution of the endocarditis findings between these groups is shown in Table 2.

The Progression Nutritional Index of the patients was lower in Group II but this did not reach statistical significance (32.4 \pm 4.5 in group I vs $29.7 \pm 4.5$ in group II, p: 0.14$)$. The Lymphocyte-CRP ratio, which was accepted as the immune nutrition score of the patients, this was significantly lower in group II $(42.4 \pm 6.8$ vs $11.3 \pm 11, \mathrm{p}: 0.04)$.

\section{Discussion}

Infective Endocarditis is a disease that is difficult to diagnose and treat. During the long treatment period, patients sometimes get into unexpected difficult situations and even lose their lives. In the recently announced Euro-Endo Study, the average age of endocarditis cases was high [9]. Especially, in patients with impaired kidney functions were shown to have a more mortal disease. In our study, kidney functions were found to be significantly impaired in our patients who died within the hospital.

In paralled with the Euro-Endo Study, the average age in our patients with endocarditis was 56 years, two-thirds of our patients were male. Especially, in the patient group where in-hospital deaths occurred LV systolic function was significantly impaired. Another difficulty of this long and rather severe infective endocarditis treatment for the patient is that, like cardiology, infection and cardiovascular surgery and when necessary other disciplines should work hand in hand with each other during the treatment process. To provide multidisciplinary approach in today's modern medical period and under increased workforce and maintaining this during the treatment process is often very difficult. However, in the multidisciplinary approach, which is carried out in difficult situations, the primary responsible cardiology physician does not lose his holistic perspective, this is perhaps the most important point that the cardiologist can provide in treatment. In patient-centered treatment, not only the biochemical and echocardiographic data of the patient but also the strength of the nutrition and immune system, which is the most important issue in the patient's removal of all this heavy treatment, should be evaluated.

The strength of the immune system associated with nurturing and nutrition is a major role modifier in an infection-based disease such as endocarditis in heart diseases. Nutrional assessment is important and for this aim, several nutrional indices have been reported to especially assess malignancy and intensive care patients. Mohri et al, found that the prognostic nutrional index has a value in predicting 
postoperative outcome after colorectal surgery [10]. Hu et all, showed that PNI may act as useful prognostic biomarkers in these patients in metaanalyses in which 15 studies were examined [11]. But, recently this issue has started to be examined in patients with heart failure and coronary artery disease $[12,13]$. Scisslo et al, have shown that patients with a poor nutrional status have a higher risk of complications after coronary artery bypass grafting operation [14]. Yoshihisa et al, found nutrional indices useful in predicting mortality in heart failure patients and PNI was superior to one of the another nutrion score 'Controlling Nutritional Status score' (CONUT) in predicting mortality among the indices [12].

However, when we look at the literature, we see indirectly the insufficiency of our holistic approach to patients in the field of modern cardiology. Especially, in the case of severe infection, such as endocarditis, the patient usually has significant weight loss due to the long time it takes for them to contact the hospital and make a diagnosis. It is the calculation of the PNI that allows us to quickly evaluate nutironal status. Practically regardless of the nutrional indexes, especially the anthropometric features of the patient. Because of this we use this index for our patient groups. On the other hand in the real cardiology practice, a cardiologist needs practical nutrional assessments, as time allocated to the patient is limited and the quality of work expected and in compliance with the guidelines at this limited time is very high. In this respect, PNI has provided us with a practical evaluation as it is quick and easy. Yoshihisa et al, patients with a PNI $>38$ are considered as normal, those with a PNI of 35-38 are at moderate risk of malnutrition and those with a PNI $<35$ are at severe risk [12]. Comparing these studies, in both groups of our patients their PNI levels are so low. Between our groups, the hospital mortality group was lower than the other but it did not reach a significant level. In addition to the CRP, which has been used recently in the evaluation of the inflammation response, it has been discussed to evaluate the immune nutrition in lymphocytes. Okugawa et al, demonstrated that this assessment of lymphocytes and CRP could aid physicians in determining the surgical and oncological risks [8] They suggested that the evaluation of the pre-operative lymphocytes to CRP ratio are clinically feasible nutrition-inflammation markers in gastric cancer patients. There is very limited information on this issue, especially in patients with endocarditis.

In a recent study by Meshaal et al, the lower lymphocyte count/percentage were strong indicators of in hospital mortality IE patients [15]. In our study, there were significantly lower lymphocyte values in patients with in-hospital mortality. However, no information is available in the literature on LCR evaluation as a nutrition-inflammation marker. In our cases LCR values were significantly lower in the group with in-hospital death. In this context we think especially in patients with low LCR values and with impaired renal function or in elderly patients, the inclusion of the nutrition team in the endocarditis multidisciplinary group during the treatment process and correcting the nutrional table that the patient needs and is missing in him will reduce the treatment complications and in-hospital mortality. In our study, there is a serious patient number limitation. However, it is important because the current disease relatively less common than other cardiac diseases and there is very limited data documented on this issue in the literature. There are no other studies in the literature that make this assessment in infective endocarditis patients. There is a need for more patient-numbered and long term evaluations of the study.

\section{Conclusion}

IE is a disease with very long and high mortality treatment. Although guidelines provide specific recommendations for treatment each patients's individual patient characteristic are different. Of these, perhaps the most neglected is nutritional status and immune resistance. In this study, it was found that patients with low Immune-Nutrional Index values had high in-hospital mortality even if they received appropriate treatment. We believe that this is an extremely important condition in patients with endocarditis.

Table 1. Comparison of basic blood values and echocardiographic dimension of patients in groups.

\begin{tabular}{|c|c|c|c|}
\hline $\begin{array}{l}\text { Characteristics and } \\
\text { Basic Findings }\end{array}$ & $\begin{array}{l}\text { Group I } \\
(n: 24)\end{array}$ & $\begin{array}{l}\text { Group II } \\
(\mathrm{n}: 12)\end{array}$ & P Value \\
\hline Age (years) & $52 \pm 15$ & $64 \pm 15$ & $0.03 *$ \\
\hline Male/Female (n) & $15 / 9$ & $9 / 3$ & 0.45 \\
\hline Hemoglobin (gr/dL) & $10.1 \pm 2.9$ & $9.7 \pm 1.6$ & 0.68 \\
\hline $\mathrm{WBC}\left(\mathrm{x} 10^{3}\right)$ & $11.6 \pm 6.9$ & $14 \pm 6.0$ & 0.2 \\
\hline Neu $\left(x 10^{3}\right)$ & $11.7 \pm 4.2$ & $11.1 \pm 3.2$ & 0.54 \\
\hline $\operatorname{Lym}\left(\mathrm{x} 10^{3}\right)$ & $1.4 \pm 0.9$ & $0.8 \pm 0.4$ & $0.02 *$ \\
\hline Platelets (x 10 $\left.0^{3}\right)$ & $274 \pm 92$ & $207 \pm 129$ & 0.19 \\
\hline CRP (mg/dL) & $90.8 \pm 81$ & $139 \pm 86$ & 0.07 \\
\hline BUN (mg/dL) & $18.2 \pm 9.5$ & $34 \pm 16$ & $0.003 *$ \\
\hline Cre $(\mathrm{mg} / \mathrm{dL})$ & $0.86 \pm 0.25$ & $1.6 \pm 1.0$ & $0.02 *$ \\
\hline ALT & $28.5 \pm 25$ & $46 \pm 13.7$ & 0.40 \\
\hline AST & $31 \pm 22$ & $72 \pm 19$ & 0.14 \\
\hline Alb (g /L) & $3.3 \pm 0.6$ & $2.97 \pm 0.4$ & 0.08 \\
\hline PNI & $32.4 \pm 6.3$ & $29.7 \pm 4.5$ & 0.14 \\
\hline $\operatorname{LCR}\left(\mathrm{x} 10^{3}\right)$ & $42.4 \pm 6.8$ & $11.3 \pm 10$ & $0.04 *$ \\
\hline LVED (mm) & $51 \pm 10$ & $57 \pm 0.8$ & $0.04 *$ \\
\hline LVES (mm) & $35 \pm 12$ & $43 \pm 11$ & $0.04 *$ \\
\hline $\mathrm{EF}(\%)$ & $50.9 \pm 13$ & $35 \pm 23$ & $0.02 *$ \\
\hline
\end{tabular}

Crp: C-reactive protein, Ast: Aspartate transaminase, Alt: Alanine aminotransferase, PNI: Progression Nutritional Index, LCR: The Lymphocyte-C-reactive Protein Ratio, LVED: Left ventricular end-diastolic dimension, LVES: Left ventricular end-systolic dimension, LVEF: Left ventricular ejection fraction

Table 2. The type of the vegetations according to groups.

\begin{tabular}{lll}
\hline & $\begin{array}{l}\text { Group I } \\
\text { (n: 24) }\end{array}$ & $\begin{array}{l}\text { Group II } \\
\text { (n: 12) }\end{array}$ \\
\hline Mitral Valve & 12 & 7 \\
Aortic Valve & 7 & 3 \\
Combination of Aortic-Mitral Valves & 4 & 2 \\
Tricuspid Valve & 1 & 0 \\
\hline
\end{tabular}

\section{References}

[1] Wang A, Gaca JG, Chu VH, Management Considerations in Infective Endocarditis: A Review. JAMA, 2018; 320 (1): 72-83. 
[2] Tleyjeh IM, Abdel-Latif A, Rahbi H, Scott CG, Bailey KR, Steckelberg JM, Wilson WR, Baddour LM, A systematic review of population-based studies of infective endocarditis. Chest. 2007; 132 (3): 1025.

[3] Selton-Suty C, Célard M, Le Moing V, Doco-Lecompte T, Chirouze C, Iung B, Strady C, Revest M, Vandenesch F, Bouvet A, Delahaye F, Alla F, Duval X, Hoen B, AEPEI Study Group. Preeminence of Staphylococcus aureus in infective endocarditis: a 1-year population-based survey. Clin Infect Dis. 2012 May; 54 (9): 1230-9.

[4] Pant S, Patel NJ, Deshmukh A, Golwala H, Patel N, Badheka A, Hirsch GA, Mehta JL. Trends in infective endocarditis incidence, microbiology, and valve replacement in the United States from 2000 to 2011. J Am Coll Cardiol. 2015; 65 (19): 2070.

[5] Habib G, Lancellotti P, Antunes MJ, Bongiorni MG, Casalta JP, Del Zotti F, Dulgheru R, El Khoury G, Erba PA, Iung B, Miro JM, Mulder BJ, Plonska-Gosciniak E, Price S, Roos-Hesselink J, Snygg-Martin U, Thuny F, Tornos Mas P, Vilacosta I, Zamorano JL; ESC Scientific Document Group. 2015 ESC Guidelines for the management of infective endocarditis: The Task Force for the Management of Infective Endocarditis of the European Society of Cardiology (ESC). Endorsed by: European Association for Cardio-Thoracic Surgery (EACTS), the European Association of Nuclear Medicine (EANM).(2015) Eur Heart J. 21; 36 (44): 3075-3128.

[6] Kono T, Sakamoto K, Shinden S, Ogawa K. Pre-therapeutic nutritional assessment for predicting severe adverse events in patients with head and neck cancer treated by radiotherapy. (2017) Clin Nutr; 36 (6): 1681e5.

[7] Onodera T, Goseki N, Kosaki G. Prognostic nutritional index in gastrointestinal surgery of malnourished cancer patients. (1984) Nihon Geka Gakkai Zasshi; 85 (9): 1001.

[8] Okugawa Y, Toiyama Y, Yamamoto A, Shigemori T, Ichikawa T, Yin C, Suzuki A, Fujikawa H, Yasuda H, Hiro J, Yoshiyama S, Ohi M, Araki T, McMillan DC, Kusunoki M. Lymphocyte-to-C-reactive protein ratio and score are clinically feasible nutrition-inflammation markers of outcome in patients with gastric cancer. (2019) Clin Nutr. 21. pii: S0261-5614 (19) 30220-1.

[9] Habib G, Erba PA, Iung B, Donal E, Cosyns B, Laroche C, Popescu BA, Prendergast B, Tornos P, Sadeghpour A, Oliver L, Vaskelyte JJ, Sow R, Axler O, Maggioni AP, Lancellotti P; EURO-ENDO Investigators. Clinical presentation, aetiology and outcome of infective endocarditis. Results of the ESC-EORP EURO-ENDO (European infective endocarditis) registry: a prospective cohort study. (2019) Eur Heart J, 40 (39): 3222-3232.

[10] Mohri Y, Inoue Y, Tanaka K, Hiro J, Uchida K, Kusunoki M. Prognostic nutritional index predicts postoperative outcome in colorectal cancer (2013) World J Surg.; 37 (11): 2688-92.

[11] Hu Y, Shen J, Liu R, Feng Z, Zhang C, Ling L, Chen L. Prognostic value of pretreatment prognostic nutritional index in non-small cell lung cancer: A systematic review and meta-analysis (2018) Int J Biol Markers, 33 (4): 372-378.

[12] Yoshihisa A, Kanno Y, Watanabe S, Yokokowa T, Abe S, Miyata M, Sato T, Suzuki S, Oikawa M, Kobayashi A, Yamaki T, Kunii H, Nakazato K, Suzuhi H, Ishida T, Takeishi Y. Impact of nutrional indices on mortality in patients with heart failure. (2018) Open Heart, 9; 5 (1): e000730.

[13] Wada H Dohi T, Miyauchi K, Endo H, Tsuboi S, Ogita M, Kasai T, Okazaki S, Isoda K, Suwa S, Daida H. Combined effect of nutritional status on long-term outcomes in patients with coronary artery disease undergoing percutaneous coronary intervention Heart Vessels. (2018) 33 (12): 1445-1452.

[14] Ścisło L, Bizoń A, Walewska E, Staszkiewicz M, Pach R, Szczepanik A. Nutritional Status of Elderly Patients after Coronary Artery Bypass Surgery. (2019) Int J Environ Res Public Health. 15; 16 (2).

[15] Meshaal MS, Nagi A, Eldamaty A, Elnaggar W, Gaber M, Rizk H. Neutrophil-to-lymphocyte ratio (NLR) and platelet-to-lymphocyte ratio (PLR) as independent predictors of outcome in infective endocarditis (IE).(2019) Egypt Heart J.; 71 (1): 13. 